\title{
Automatic detection of crystallographic defects in STEM images by unsupervised learning with translational invariance
}

Yueming Guo ${ }^{1}$, Andrew R. Lupini ${ }^{2}$, Hui $\mathrm{Cai}^{3}$, Kai Xiao ${ }^{2}$, Sergiy Krylyuk ${ }^{4}$, Albert Davydov ${ }^{4}$, Qianying Guo ${ }^{2}$ and Sergei Kalinin ${ }^{5}$

${ }^{1}$ Oak Ridge National Laboratory, OAK RIDGE, Tennessee, United States, ${ }^{2}$ Oak Ridge National Laboratory, United States, ${ }^{3}$ University of California, Merced, United States, ${ }^{4}$ National Institue of Standards and Technology, United States, ${ }^{5}$ Center for Nanophase Materials Sciences, Oak Ridge National Laboratory, Oak Ridge, Tennessee, United States

Crystallographic defects are interruptions of periodic patterns in crystals of real materials. Since a human scientist with some basic knowledge of crystallography is easily able to recognize the defects from atomic-resolution images, it is not obvious to realize how difficult it is for a computer code to identify crystallographic defects from a sequence of numbers representing intensities. Like many other visual intelligence tasks for identifying daily objects, identification of crystallographic defects is a challenging issue and it is more challenging when no human-labeled examples of the defects are given to train the model before the identification, i.e. in unsupervised learning.

Supervised learning methods based on artificial neural networks (NNs) have previously been applied to identification of crystallographic defects [1,2]. Either experimental or simulated images of the defects are used to train the NNs, which are then applied to identify defects from new datasets. Although these methods have been successful in handling specific examples of defects in the crystal structures being investigated, the trained NNs are usually limited to the crystal structure in the training set and cannot easily be generalized to other crystal systems. In addition, labelling of defects by hand can become very costly when the crystal structure is complex and possible configurations of the defects are highly diverse. Apart from supervised machine learning, unsupervised detection (i.e. without labelling) of crystallographic defects has been approached by explicitly programing a set of rules derived from graph theory [3]. However, this approach is more time-consuming in the implementation stage and would become less efficient with increasing number of atoms in the unit cell. So far, a really reliable unsupervised machine learning method for automatic detection of crystallographic defects in STEM images is lacking.

We present an unsupervised learning based method for detecting crystallographic defects in STEM images. The method consists of a one-class support vector machine (OCSVM) [4], new schemes of image segmentation, and a translational-invariant descriptor based on the Patterson function. Two examples are demonstrated: the first example is $2 \mathrm{H}$ phase $\mathrm{MoWTe}_{2}$ with beam-induced defects; the second example is a $\mathrm{ZrO}_{2}$ nanoparticle with a twin boundary. Locations with misplaced or missing atoms are identified in the first example and the existence of two different domains is identified in the second example. With the new scheme of image segmentation used in the second example, our method may also achieve unsupervised classification of multiples phases in a STEM image. [5] 


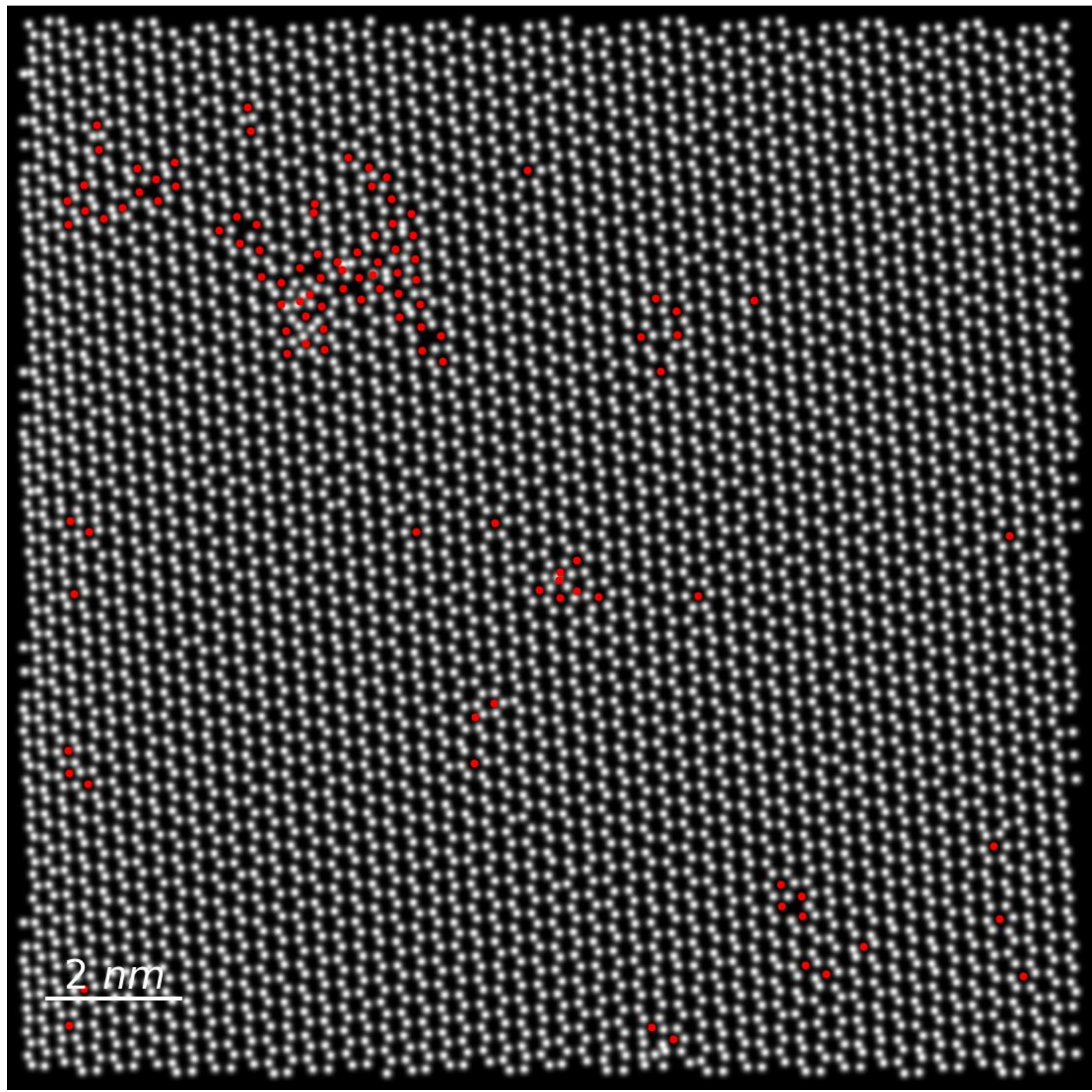

Figure 1. The result of detecting defects where atoms are misplaced or missing. The defects are labelled with red dots in a semantic image of a bilayer $2 \mathrm{H}$ MoWTe2. The semantic image does not carry the information about the atom species but only the positions of atom columns. The original STEM image was recorded with NION UltraSTEM 200 at $100 \mathrm{kV}$. 


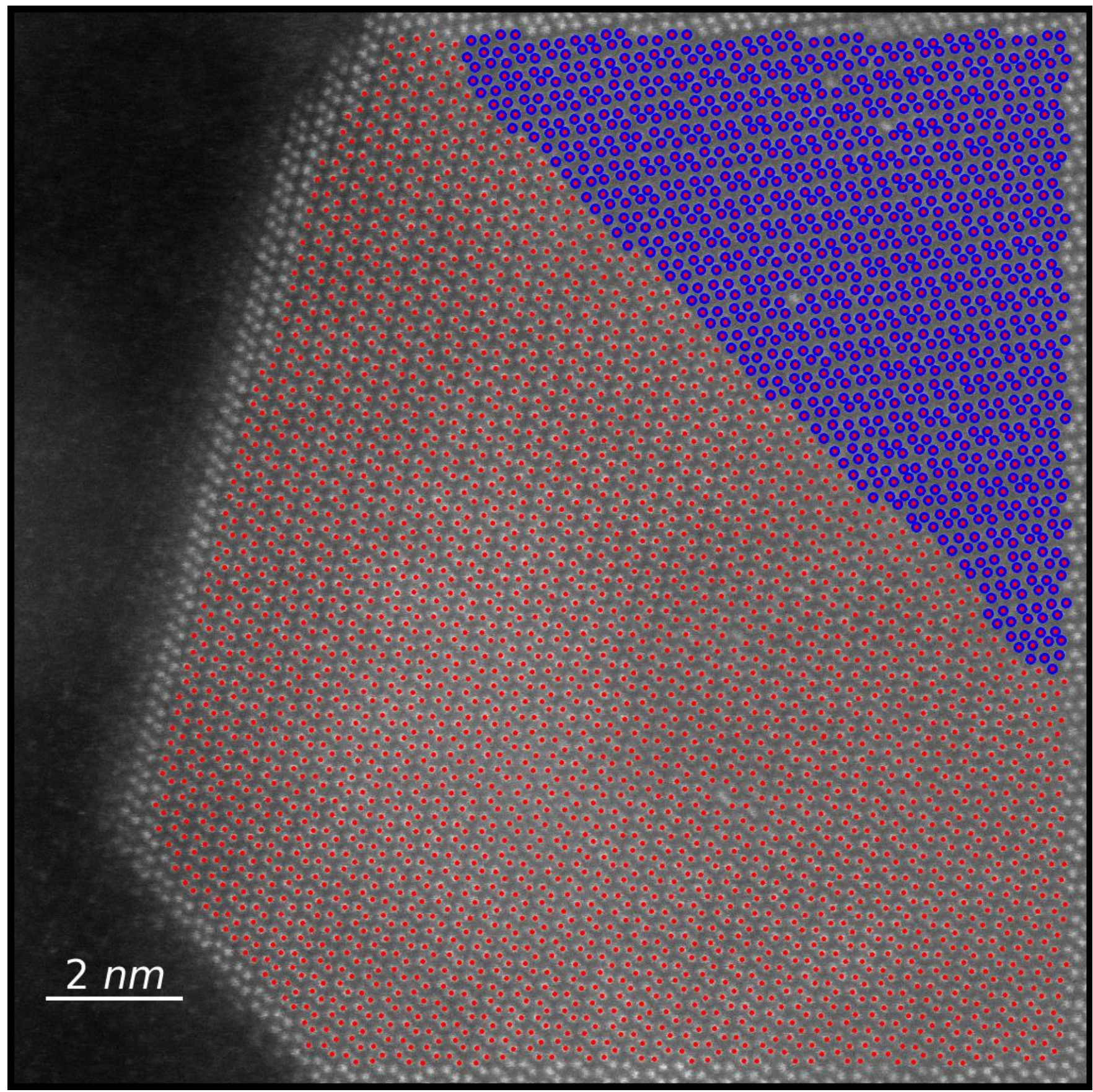

Figure 2. The result of detecting the existence of two domains on each side of a twin boundary in a monoclinic $\mathrm{ZrO} 2$ nanoparticle. The STEM image was recorded with NION UltraSTEM 100 at $100 \mathrm{kV}$.

\section{References}

[1] M. Ziatdinov et al., Acs Nano 11, 12742 (2017).

[2] C. H. Lee et al., Nano Lett 20, 3369 (2020).

[3] O. S. Ovchinnikov et al., Adv Struct Chem Imag 6 (2020).

[4] B. Scholkopf, R. Williamson, A. Smola, J. Shawe-Taylor, and J. Platt, Adv Neur In 12, 582 (2000).

[5] Work supported by the U.S. Department of Energy (DOE), Office of Science, Basic Energy Sciences (BES), Materials Sciences and Engineering Division, and performed at the Center for Nanophase Materials Sciences (CNMS), a U.S. Department of Energy Office of Science User 